\title{
A Quantitative Vascular Analysis System for Evaluation of Atherosclerotic Lesions by MRI
}

\author{
William Kerwin ${ }^{1}$, Chao $\mathrm{Han}^{1}$, Baocheng $\mathrm{Chu}^{1}$, Dongxiang $\mathrm{Xu}^{2}$, Ying Luo ${ }^{2}$, \\ Jenq-Neng Hwang ${ }^{2}$, Thomas Hatsukami ${ }^{3}$, and Chun Yuan ${ }^{1}$ \\ 1 University of Washington, Department of Radiology, Box 357115, 1959 NE Pacific \\ St., Seattle, WA 98195, USA \\ \{bkerwin, chaohan, chubc, cyuan\}@u . washington.edu \\ http://vil.rad.washington.edu \\ 2 University of Washington, Department of Electrical Engineering, Box 352500, \\ Seattle, WA 98195-2500, USA \\ $\{\mathrm{xdx}$, luoying, hwang $\}$ @ee . washington.edu \\ 3 University of Washington, Department of Surgery, Box 358280, 1660 S Columbia \\ Way, Seattle, WA 98108, USA \\ tomhat@u.washington. edu
}

\begin{abstract}
An analysis package called QVAS (quantitative vascular analysis system) is presented for the evaluation of atherosclerotic arterial lesions visualized in vivo by magnetic resonance imaging. QVAS permits interactive identification of vessel and lesion boundaries, segmentation of tissue classes within the lesion, quantitative analysis of lesion features, and three dimensional display of lesion structure. The performance of QVAS is demonstrated using images of carotid artery lesions.
\end{abstract}

\section{Introduction}

Cardiovascular disease is the leading cause of death in the West and most heart attacks and strokes result when an atherosclerotic lesion ruptures, which leads to local formation of a thrombus or release of emboli that block distal vessels [1]. The structural features that predispose lesions to rupture can now be resolved in vivo using high resolution magnetic resonance (MR) imaging, at least for large vessels such as the carotid arteries 23. From MR images, quantitative measurements of the lesion size, composition, and distribution can be made, which may allow researchers to study lesion progression, pharmacologists to evaluate the response to drug therapy, or clinicians to assess the risk associated with a particular lesion [4,5]. Such analyses would be facilitated by a computerbased package for the evaluation of vascular MR images.

A number of general purpose packages for the evaluation of medical images exist, including NIH Image (National Institutes of Health, Bethesda, MD) and ANALYZE (Mayo Clinic, Rochester, MN). For specialized applications, however, these packages are often insufficient. As a result, tailored image analysis packages have been proposed, including MASS and FLOW (MEDIS, Danbury, CT), which are used for quantitative measurement of cardiac function. A similar package 
specifically designed for analysis of atherosclerotic lesions is warranted by several challenging aspects of the lesions. First, the lesions are small, with diameters on the order of 1-2 cm at the largest. Within this small space, lesions often exhibit a highly complicated morphology with rapid transitions between tissue types. Finally, any of a large number of clinically significant tissue types may or may not be present in a given lesion.

In this paper we present a comprehensive, tailored analysis package for evaluating atherosclerotic lesions as visualized by MR imaging. The package is called the Quantitative Vascular Analysis System (QVAS) and addresses three primary goals. Foremost, QVAS enables computer assisted identification of lesion boundaries, constituent tissue types, and distribution of tissues. Second, the information extracted via QVAS can be converted into quantitative measurements of lesion severity. Third, QVAS permits the construction of a three dimensional model of the lesion for use in an interactive display.

The current version of QVAS is capable of processing a single set of MR images that span the length of a lesion and have a single contrast weighting. Individual images are serially processed to identify relevant boundaries and tissue types. Extracted information is then propagated from one image to the next to increase automation.

This paper is organized as follows. In Section 2, we describe the pathology of atherosclerotic lesions and their visualization by MR imaging. Then, in Section 3 , we provide a synopsis of the image processing algorithms utilized by QVAS. In Section 4, we discuss data flow and demonstrate it with results from carotid artery lesions. Finally, we conclude in Section 5 with a discussion of QVAS applications and planned improvements to QVAS.

\section{Background on Atherosclerosis}

Pathology. A typical advanced atherosclerotic lesion surgically removed from a carotid artery is shown in Fig. 13. At the center is the lumen, which is the normal passageway for blood. Separating the lumen from the bulk of the lesion is an encapsulating fibrous layer (fibrous cap). Behind the cap lies the core of the lesion, which may include lipid-rich necrotic material, cholesterol crystals, calcifications, intraplaque hemorrhage, and/or microvessels.

Culprit lesions, implicated in heart attacks, are characterized by erosion and rupture of the fibrous cap exposing the thrombogenic core 6]. Traditionally, the risk of rupture is determined by percent narrowing of the artery (stenosis), but lesions with only moderate stenosis often rupture as well [7]. Studies show that the fibrous cap may be prone to rupture if it is markedly thinned, overlies a predominantly lipid core, is adjacent to substantial amounts of microvessels, or has adjacent calcifications [1,6,8]. Identification of these lesion features may therefore provide the best risk assessment.

Imaging. Magnetic resonance imaging has been shown to be sensitive to the different tissues present in atherosclerotic lesions and therefore can be used to 

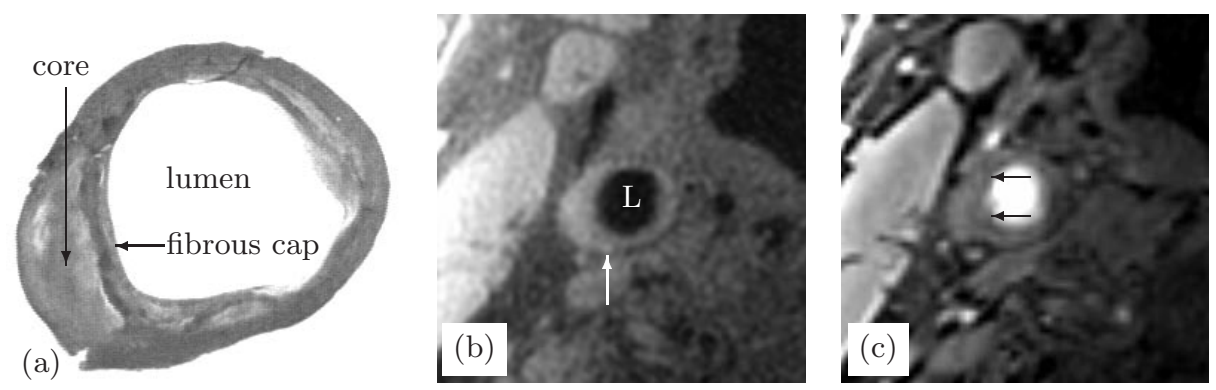

Fig. 1. Atherosclerotic lesion: (a) surgically removed from a carotid artery and stained (H\&E), with tissue regions labeled; (b) imaged in vivo (vessel indicated by arrow, lumen by "L") using a black-blood double inversion recovery $\mathrm{T} 1$ weighted sequence $(\mathrm{TR} / \mathrm{TI} / \mathrm{TE}=800 / 650 / 9 \mathrm{msec})$; (c) imaged in vivo using a time-of-flight sequence $(\mathrm{TR} / \mathrm{TE}=23 / 4 \mathrm{msec})$ that generates a bright lumen and dark fibrous cap (arrows).

assess risk of rupture [3]. Most in vivo studies have involved the relatively large carotid artery, which is commonly implicated in strokes and can be imaged in high detail because its location in the neck permits the use of highly sensitive surface coils [29]. Other studies have shown the potential for MR imaging of aorta lesions and coronary lesions [10,11].

Typical in vivo images of a carotid lesion are shown in Fig. 1 with T1 and time-of-flight (TOF) contrast weightings. The T1 weighted image provides the best contrast for the lumen and outer wall boundaries. The TOF weighting provides the best visualization of the fibrous cap, which is characterized by low intensity 4. Lipid-rich core and hemorrhage are both characterized by moderate to high intensities on T1 and TOF weighted images. Calcifications are characterized by low intensity on all contrast weightings.

\section{Image Processing Methods}

The fundamental processing goal for quantitative analysis of lesion structure is to divide the MR images into regions of known tissue type. Our framework for this division is a "coupled contour mesh" as illustrated in Fig. 2. The coupled contour mesh consists of "nodes," denoted by the asterisks, and "paths" that connect the nodes. A region is delineated by a closed sequence of nodes and the paths connecting them. Finally, each region may have a corresponding tissue type if it is known. Generating such a coupled contour mesh is accomplished interactively with the assistance of several basic image processing tools: segmentation, boundary identification, and tissue classification.

Segmentation. The first image processing tool we employ segments the image pixels into clusters with similar brightness characteristics. The segmentation 

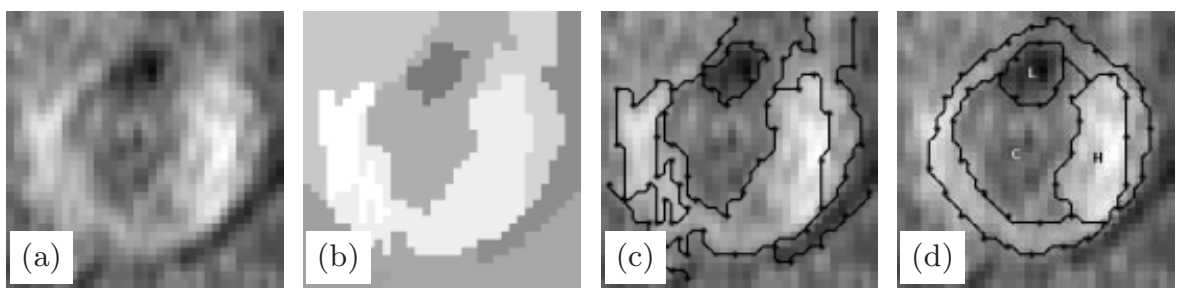

Fig. 2. Processing of a coupled contour mesh: (a) Region of interest around an atherosclerotic lesion; (b) Segmented image with different regions shown as different gray scales; (c) Coupled contours that divide the image into the segmented regions; (d) Coupled contours after editing with the active contour algorithm and tissue classification ( $\mathrm{L}=$ lumen, $\mathrm{C}=$ core, $\mathrm{H}=$ hemorrhage).

algorithm is a two step Markov random field (MRF) approach called quadtree highest confidence first (QHCF) [12. The first step in QHCF is to identify $m$ seed pixels as initial estimates of cluster means and locations. This is done by quadtree division of an image into rectangular subregions until the variance of all pixel intensities within each subregion falls below a user-specified threshold $T$. Then, the one pixel in each subregion that is closest to the mean intensity is extracted and used as an initial cluster, while all other pixels are left unassigned.

The final clusters are identified by seeking the maximum of an MRF probability function or, equivalently, by minimizing

$$
E=\sum\left[\left(y_{i}-\mu_{i}\right)^{2}+V_{N}\left(y_{i}\right)+V_{E}\left(y_{i}\right)\right],
$$

where $y_{i}$ is the intensity of the $i^{\text {th }}$ pixel, $\mu_{i}$ is the mean of the region to which the $i^{\text {th }}$ pixel has been assigned, and $V_{N}$ and $V_{E}$ are additional energies based on neighborhoods and edges, respectively. The term $\left(y_{i}-\mu_{i}\right)^{2}$ forces pixels to be assigned to a region with similar intensities. The term $V_{N}$ favors assignments to the dominant region in a $3 \times 3$ neighborhood $N$ and is given by

$$
V_{N}\left(y_{i}\right)=\beta_{1} \sum_{j \in N} U(i, j),
$$

where $U(i, j)$ is 1 if $y_{i}$ and $y_{j}$ are in different regions and -1 if they are in the same region; $\beta_{1}$ is a user-specified weight. The term $V_{E}$ adds an additional penalty if pixels on opposite sides of a Canny-detected edge are assigned to the same region. Finally, the assignments to regions are performed by iteratively finding the pixel assignment that will provide the greatest reduction in (11) until no further reductions are possible, an approach known as "highest confidence first." An example of a segmented image is shown in Fig. 2b.

Active Contour Boundary Identification. From the segmentation, a coupled contour mesh can be generated, as in Fig. 22k, by placing nodes at intersections of three or more regions and at intervals along boundaries between two regions, 
and then connecting the nodes with paths running along region boundaries. To correctly identify tissue regions, however, this mesh requires further editing, including insertion of nodes and paths to define or split regions, removal of nodes and paths to combine regions, and repositioning of existing nodes. After manually editing node positions, new paths between connected nodes are generated with an active contour method that searches for a minimal energy path 13 .

The minimal energy path between fixed nodes is defined as the curve $C(v)$ that minimizes

$$
E=\int_{\Omega} g(|\nabla I(C(v))|)\left|C^{\prime}(v)\right| d v
$$

where $\nabla I$ is the gradient of the image, $g$ is a strictly decreasing potential and $\Omega \in[0,1]$ is the parameterization interval for the contour segment. Based on this formulation, the minimal path will follow high gradient boundaries in the image and will be smoothly varying so that the first derivative is near zero. In practice, this minimal path is approximated by selecting a sequence of points from one node to the other, where the optimal sequence of points is found using graph search techniques over a finite grid. An example of the end result of boundary identification is shown in Fig. 2 d.

The search for a minimal path does not address the fact that the original nodes may not have been placed at minimal energy positions. The QVAS package therefore also includes two optional contour evolution modes in which selected node positions are updated. The first is known as "wriggling," which progressively refines overlapping subsegments of a contour that may include nodes [13. The second mode is "rule-based wriggling," which seeks a specific tissue type within a closed contour. In this mode, a contour segment will balloon outward if the adjacent pixels on either side have a mean intensity that is similar to the desired tissue (i.e. the segment lies within the tissue). The segment shrinks inward if the adjacent pixels on either side have a mean intensity that is not similar (i.e. the segment is outside the tissue). Finally, the standard minimal path search is employed if only the pixels on the inner side of the segment are similar in mean intensity to the desired tissue (i.e. the segment is near the boundary). If the initial boundary contains any portion of the desired tissue within it, then rule-based wriggling will converge to the correct boundary, even if the initial estimate of contour position is poor.

Tissue Classification Once the tissue regions have been identified, a tissue label such as fibrous cap, lipid core, or calcification must be associated with each. In general, tissue labeling must be done manually by a trained expert. We have, however, developed an adaptive algorithm that facilitates labeling. For each contrast weighting, we have compiled a library of regional brightness values and associated tissue types. To assign a label to an unknown region we find the tissue type from the library with the nearest mean brightness value. To account for absolute signal differences, we perform histogram equalization before computing means or making comparisons.

Initially, this labeling scheme is highly prone to errors because of the variation between patients. As the QVAS user corrects mislabeled regions by hand, the 
program enters an adaptive mode, wherein the mean intensities from the library are adjusted to better match the current lesion. The adjustment is performed along the first two principle components of variation, seeking to minimize the total squared difference between the adjusted means and the brightness values observed in the current lesion. As QVAS adapts in this manner, fewer and fewer user interventions are necessary.

\section{Image Analysis Results}

Building a Coupled Contour Typical steps by which a QVAS user employs the image processing tools to construct a coupled contour mesh are illustrated in Fig. 3. First, a region of interest is identified around the artery (Fig. 3 a) and the segmentation algorithm is applied. The threshold $T$ is interactively adjusted until a small number of regions approximately covers the vessel lumen and an additional set covers the entire vessel wall (Fig. 3 b). Then the sets of nodes that define the lumen and outer wall boundaries are chosen (Fig. 35), which provides an initial delineation of the lesion boundaries. Next, refinement of the lesion boundaries is performed using the active contour algorithm (Fig. 3 $\mathrm{d}$ ). Ordinarily, the wriggle procedure is used to automatically adjust the boundaries to minimum energy positions. If, however, the boundary is partially obscured or otherwise poorly defined, the user may be required to manually adjust the nodes of one or both contours. Once the lesion boundaries have been finalized, the segmentation algorithm is reapplied, this time only to the pixels contained within the contours. Again, the user interactively controls the segmentation threshold, selecting a level that best delineates the observed regions in the lesion (Fig. 3. ). Finally, the subregions are manually edited using the active contour tools and semi-automated tissue classification is performed (Fig. 3f).

Feed Forward To characterize an entire lesion volume, coupled contour meshes must be built for each of a stack of images spanning the lesion. Although each image could be treated independently, significant time savings are possible if information is fed forward from one image to the next using the following hierarchy. First, the lumen and outer wall boundaries from the previous image are mapped to the next image and allowed to evolve by wriggling, specifically using rule-based wriggling for the more homogeneous lumen. If either boundary search fails, user adjustment is necessary. Next, the segmentation algorithm is applied seeking initial quadtree regions with intensities similar to the previous regions. After application of the segmentation algorithm, the user may manually adjust the delineated regions. Alternatively, if the segmentation results are poor, the algorithm may be reapplied using the standard quadtree initialization scheme. Finally, for tissue classification, the adapted tissue means from the previous image are used in the current one. The end result is a complete $3 \mathrm{D}$ segmentation of the lesion, an example of which is rendered in Fig 4 

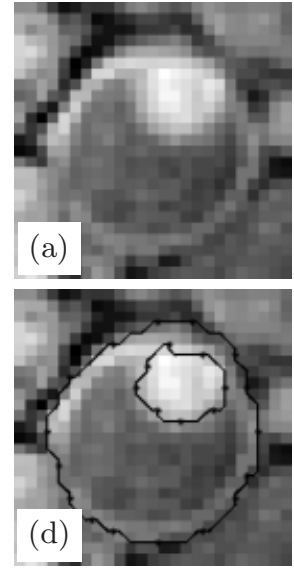
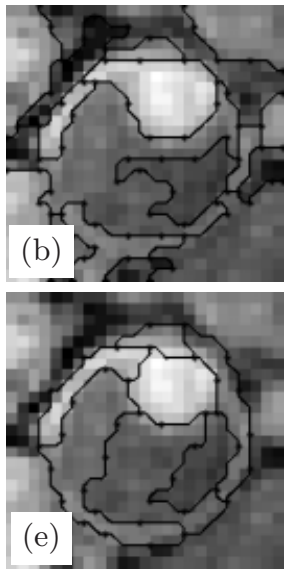
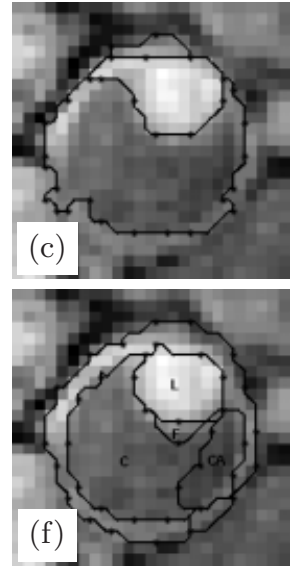

Fig. 3. Processing steps in lesion analysis: (a) region of interest is selected; (b) segmentation algorithm is applied; (c) contours that best correspond to the lumen and outer wall boundaries are chosen; (d) boundaries are refined using the active contour algorithm; (e) regions between the boundaries are segmented; (f) regions are edited and assigned tissue types: lumen (L), fibrous (F), core (C), calcium (CA).

\section{Discussion}

The QVAS package is an interactive environment for rapid processing of MR images of atherosclerotic lesions to identify tissue regions and types for clinical and investigative applications. Specifically, QVAS facilitates quantitative measurements such as total volume, percent lipid content, or fibrous cap thickness. Such measurements may be formulated as a "lesion index" for monitoring response to therapy or assessing risk of heart attack or stroke [14].

Additionally, the regions identified by QVAS can be used for volumetric reconstruction of the lesion, which may be used to study disease progression or for surgical planning. For example, the artery reconstructed in Fig. 4 was imaged prior to endarterectomy. The lumen size (Fig. 4 4 ) is ordinarily the only information available to the surgeon via angiography. While this clearly indicates the presence of a large lesion impinging on the internal carotid artery, it does not show that significant calcifications exist in the vicinity of the bifurcation, as seen in Fig. 4b. QVAS thus provides important information for surgical planning.

Finally, although QVAS incorporates a number of automatic and semiautomatic tools for the user, further automation would be necessary for routine clinical use. Also, simultaneous analysis of multiple images taken at the same position is necessary for applications such as the study of contrast agent dynamics. For these reasons, we are developing an advanced version of QVAS that simultaneously operates on multiple images of the same slice obtained with different contrast weightings. The package will include a registration module, a multispectral segmentation algorithm, and an active contour model that com- 


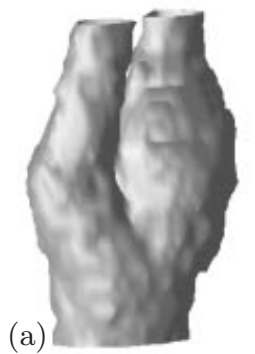

(a)

(b)

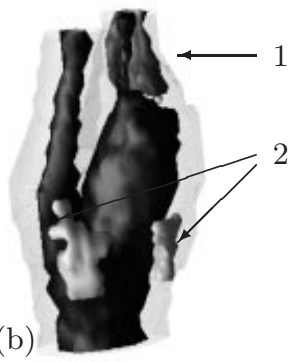

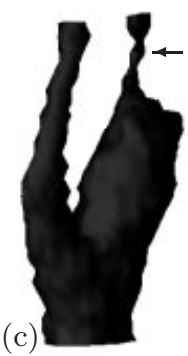

Fig. 4. Reconstructed carotid arteries rendered using OpenDX (IBM, Armonk, NY): (a) Outer wall of the carotid arteries showing the common carotid artery bifurcating into the external carotid artery (in front) and internal carotid artery (in back); (b) Within the wall, a lipid core (1) and several calcifications (2) are present; (c) The severely stenosed (arrow) vessel lumen shows evidence of the lipid core, but no indication of the calcifications.

bines boundary information from multiple images. The details of the new version will be reported in the future.

Acknowledgement. This work was supported by NIH grant R01-HL61816 and by AstraZeneca pharmaceuticals.

\section{References}

1. Fuster, V., Stein, B., Ambrose, J.A., et al.: Atherosclerotic plaque rupture and thrombosis. Evolving concepts. Circ. 82(3 Suppl) (1990) II47-59

2. Fayad, Z.A., Fuster, V.: Characterization of atherosclerotic plaques by magnetic resonance imaging. Ann. N.Y. Acad. Sci. 902 (2000) 173-186

3. Ingersleben, G.V., Schmiedl, U.P., Hatsukami, T.S., et al.: Characterization of atherosclerotic plaques at the carotid bifurcation: Correlation of high resolution MR with histology. RadioGraphics 17 (1997) 1417-1423

4. Hatsukami, T., Ross, R., Polissar, N., Yuan, C.: Visualization of fibrous cap thickness and rupture in human atherosclerotic carotid plaque in vivo with high resolution magnetic resonance imaging. Circ. 102 (2000) 959-964

5. Yuan, C., Beach, K.W., Smith, H.L., Hatsukami, T.: In vivo measurements of maximum plaque area based on high resolution MRI. Circ. 98 (1998) 2666-2671

6. Falk, E.: Why do plaques rupture? Circ. 86 (6 Suppl) (1992) III30-42

7. Libby, P.: The interface of atherosclerosis and thrombosis: Basic mechanisms. Vasc. Med. 3 (1998) 225-229

8. Virmani, R., Kolodgie, F.D., Burke, A.P., et al.: Lessons from sudden coronary death. A comprehensive morphological classification scheme for atherosclerotic lesions. Aterioscler. Thromb. Vasc. Biol. 20 (2000) 1262-1275

9. Hayes, C.E., Mathis, C.M., Yuan, C.: Surface coil phased arrays for high resolution imaging of the carotid arteries. JMRI 1 (1996) 109-112

10. Fayad, Z.A., Nahar, T., Fallon, J.T., et al.: In vivo magnetic resonance evaluation of atherosclerotic plaques in the human thoracic aorta. Circ. 101 (2000) 2503-2509 
11. Fayad, Z.A., Fuster, V., Fallon, J.T., et al.: Noninvasive in vivo human coronary artery lumen and wall imaging using black-blood magnetic resonance imaging. Circ. 102 (2000) 506-510

12. Xu, D., Hwang, J.N., Yuan, C.; A robust method of identifying and measuring fibrous cap in 3D time-of-flight MR image. Proc. ICIP 2 (1999) 164-167

13. Han, C., Hatsukami, T.S., Hwang, J.N., Yuan, C. A fast minimal path active contour model. IEEE Trans. Image Proc. (to appear)

14. Yuan, C., Kang, X., Xu, D., Hatsukami, T.S.: Lesion index: A quantitative measure of atherosclerotic lesion complexity and progression in MR images. Proc. ISMRM (1999) \#85 